\title{
INFLUENCIA DE TÉCNICAS DE MEJORA DE SUELOS SOBRE LA FUNCIÓN HÍDRICA DE PASTOS NATURALES ALTOANDINOS
}

\section{INFLUENCE OF SOIL IMPROVEMENT TECHNIQUES ON WATER FUNCTION OF ANDEAN RANGELANDS}

\author{
Luis Oscanoa ${ }^{1}$ y Enrique Flores ${ }^{2}$
}

\begin{abstract}
Resumen
Se investigaron los pastos naturales degradados de la comunidad campesina Cordillera Blanca, Huaraz - Perú. El objetivo fue estudiar la influencia de las técnicas de mejora (T) sobre el estado ecológico (Ee), recuperación hidrológica $(\mathrm{H})$ y control de la erosión (Er) del pastizal de condición muy pobre. El diseño experimental fue completamente al azar con arreglo factorial (3 técnicas x 6 épocas por parámetro de estudio). La investigación duró dos años, etapa en la cual se estudiaron el Ee (vegetación, biomasa y mantillo), H (infiltración, humedad y temperatura) y Er. Los resultados indicaron que a corto plazo la condición muy pobre del pastizal no cambia, pero si los demás parámetros. En época de lluvias del año dos, las parcelas con surcos alcanzaron mejor rendimiento vegetal que hoyos y uso comunal (biomasa aérea: 1 755, 1562 y 1067 kg ms/ha; mantillo: 402, 388 y 305 kg ms/ha, respectivamente). El mismo orden presentó la respuesta hidrológica a través del parámetro tasa de infiltración $(0.120,0.110$ y $0.065 \mathrm{~cm} / \mathrm{min})$, contenido de humedad (20.8, 20.4 y 16.6\%) y temperatura del suelo $\left(11,10.7\right.$ y $\left.10^{\circ} \mathrm{C}\right)$. Los resultados sugieren que la mejora florística y función hidrológica habrían coadyuvado a controlar la erosión del suelo en parcelas tratadas con surcos y hoyos (36 y 45 ton/ha/2años) que el uso comunal (58 ton/ha/2años). Se concluye que la recuperación del pastizal pobre es lenta; la humedad, biomasa e infiltración es de alta sensibilidad; y la técnica de surcos y hoyos son opciones accesibles para la mejora del pastizal degradado. Se recomienda estudiar la interacción humedad del suelo con las especies vegetales claves para la mejora ecohidrológica de los tipos de pastos naturales altoandinos.
\end{abstract}

Palabras clave: Pastos naturales, función hídrica, surcos, hoyos, control erosión.

\begin{abstract}
Research was performed in degraded native grasslands in the rural community of Cordillera Blanca, Huaraz _ Peru. The objective was to study the influence of improvement techniques (T) on the ecological status (Ee), hydrological recovery $(\mathrm{H})$ and erosion control $(\mathrm{Er})$ of very poor condition grasslands. The experimental design was completely randomized with a factorial arrangement of 3 techniques x 6 seasons sampling by study parameter. The Ee (vegetation, biomass and mulch), $\mathrm{H}$ (infiltration, soil moisture and temperature) and Er were studied during the two years of research. Results showed that in the short term the poorest range condition did not change, except in the other parameters. Rainy season plots with furrows got better plant performance than pits and community use (aerial biomass: 1755, 1562 and $1067 \mathrm{~kg} \mathrm{dm} / \mathrm{ha}$; mulch: 402, 388 and $305 \mathrm{~kg} \mathrm{dm} / \mathrm{ha}$, respectively). The same order was observed in the hydrological response through the soil temperature parameter $\left(11,10.7\right.$ y $\left.10^{\circ} \mathrm{C}\right)$, moisture content $(20.8,20.4 \mathrm{y}$ $16.6 \%)$ and infiltration rate $(0.120,0.110$ y $0.065 \mathrm{~cm} / \mathrm{min})$. The results suggested that the improvement in floristic and hydrological function must have controlled the soil erosion in plots treated with grooves and pits. We conclude that poor grassland recovery is slow; soil moisture, biomass and infiltration is highly sensitive; and technical groove and pits are accessible options for improving degraded grasslands. It is recommended to study the interaction of soil moisture with plant species for the ecohydrological improvement of Andean rangeland types.
\end{abstract}

Key words: Andean rangelands, water function, furrows, pits, control erosion.

\section{Introducción.}

En el Perú, del total de pastos naturales, los pastizales de condición pobre y muy pobre ocupan el 64\% de la superficie (Florez \& Malpartida, 1987), situación que refleja inadecuada utilización del pastizal debido al limitado conocimiento integral de la interacción clima, hidrología, vegetación y socio economía rural (Getahun et al., 2014). Se predice que 
el cambio climático incrementará la escorrentía, erosión del suelo y el empobrecimiento de los pastizales, por lo que la gestión de recursos amerita adaptarse al aumento del riesgo de nuevos escenarios (Zhang et al., 2012). En estos últimos tiempos renace una sociedad más sensible al problema del agua, y los proyectos de montañas re-direccionan sus acciones encaminadas a captar el agua de lluvias a través del uso racional del ecosistema pastizal, pero aún con discrepantes intervenciones.

En cuencas altas donde predominan las condiciones climáticas extremas, escasez de agua, erosión y presencia de pastos nativos, es muy importante conocer la función hidrológica de las gramíneas sin importar el origen biogeográfico (Viramontes-Olivas et al., 2012). La producción de forraje de pastizales de secano durante la estación de crecimiento depende de la precipitación (Bork et al., 2001); de manera que el clima influye en la variación de la productividad primaria neta del pastizal que trasciende en aumento de biomasa durante la época húmeda y disminución en épocas áridas (Ma et al., 2010). En la tundra alpina, el clima, la humedad del suelo y la composición química de la hojarasca son variables principales para la descomposición, ciclo de nutrientes, velocidad y transferencia de carbono fijado en los depósitos de materia orgánica del suelo de los ecosistemas terrestres (Bryant et al., 1998).

Las especies vegetales del pastizal afectan diferenciadamente el desarrollo de los parámetros de la comunidad vegetal, y debido a que las correlaciones entre las características son débiles y cambiantes con la estación de crecimiento y época del año, fue más útil considerar los atributos biomasa aérea, mantillo, humedad del suelo y temperatura del suelo de forma independiente (Eviner, 2004). De manera que los tipos funcionales de plantas, en ecosistemas semiáridos, son afectados por la distribución de agua disponible en el suelo y las relaciones subyacentes entre la heterogeneidad de humedad del suelo y la composición del pastizal, por lo que éstas son la base para la predicción de las interrelaciones entre los procesos hidrológicos y la dinámica de la vegetación (Breshears \& Barnes, 1999).

Parámetros del suelo, como la humedad del suelo, muy pocas veces fueron tomados en cuenta para evaluar la condición del pastizal y la autorregulación biótica del ecosistema pastizal (Eekeren et al., 2010). Las zanjas de infiltración son una alternativa efectiva para el control de la erosión y la captación de agua de lluvia en laderas (Vásquez \& Tapia, 2011), pero estos aún con muy poca réplica a nivel del pequeño productor. En este contexto, los pastizales pobres son recursos frágiles donde se asientan sistemas pobres de crianza mixta y animales silvestres; de ahí la necesidad de construir opciones que mejoren este territorio con la finalidad de controlar la erosión del suelo, el balance hídrico y reducir la pobreza de las familias ganaderas.

El objetivo del presente estudio fue investigar la influencia de las técnicas de mejora (surcos y hoyos) sobre el estado ecológico (vegetación, biomasa y mantillo), función hidrológica (infiltración, humedad y temperatura) y erosión de suelos cubierto de pastos naturales altoandinos de condición muy pobre. Las preguntas de investigación fueron: ¿Cuál es el nivel de interacción entre las técnicas de mejora de suelos y épocas del año?, ¿Cuál es el parámetro de mayor relevancia para el estudio ecohidrológico del pastizal?, ¿En qué medida la condición muy pobre del pastizal se recupera con las técnicas de mejora surcos y hoyos?

\section{Materiales y métodos.}

Área experimental

La presente investigación se realizó desde mayo 2011 a setiembre 2013 en pastos naturales de la comunidad campesina Cordillera Blanca - Huaraz, Perú a una altitud de 4100 msnm (UTM 238420.85 8928842.54). El área experimental, según Holdridge (1987) se clasificó como Páramo muy húmedoSubalpino Tropical (Pmh-SaT) con temperatura promedio anual de $12^{\circ} \mathrm{C}$, y una precipitación promedio anual de $1318 \mathrm{~mm}$ (Gil, 2014). El territorio experimental se encuentra dominado por la asociación vegetal Calamagrostis macrophylla - Stipa brachyphylla.

El estado del pastizal fue de condición muy pobre con tendencia negativa para ovinos, vacunos y vicuñas. Los suelos son de característica pedregosa, textura franco arenoso y con rasgos de erosión laminar. El análisis de suelo muestra un $\mathrm{pH} 4.53$, conductividad eléctrica $0.11 \mathrm{dS} / \mathrm{m}$, capacidad de intercambio catiónico $9.12 \mathrm{meq} / 100 \mathrm{~g}$, contenido de fósforo $3.4 \mathrm{ppm}$, potasio $24 \mathrm{ppm}$ y materia orgánica de 4.2 \% (Laboratorio de Análisis de Suelo y Aguas UNALM, 2011).

El sitio experimental "Sillacancha" pertenece a la cadena de cabeceras de cuenca del rio Santa y forma parte de un estudio más amplio sobre recuperación del ecosistema pastizal altoandino degradado (Figura 1). El pastoreo de éstas es de forma tradicional, manejadas extensivamente por grupos de familias socias de la comunidad campesina. El movimiento animal de las partes altas a las bajas es forzado debido a la presencia de rayos durante la época de lluvias que provocan mortalidad animal y humana. La construcción de cercos de uso agroforestal y zanjas de infiltración es creciente, los cuales afectan el flujo técnico de programas de manejo del hato ganadero. La selección del área experimental, diseño y monitoreo de los parámetros se realizaron participativamente con el Comité de Investigación Agropecuaria Local CIAL “Alli Pastu” de la comunidad. 


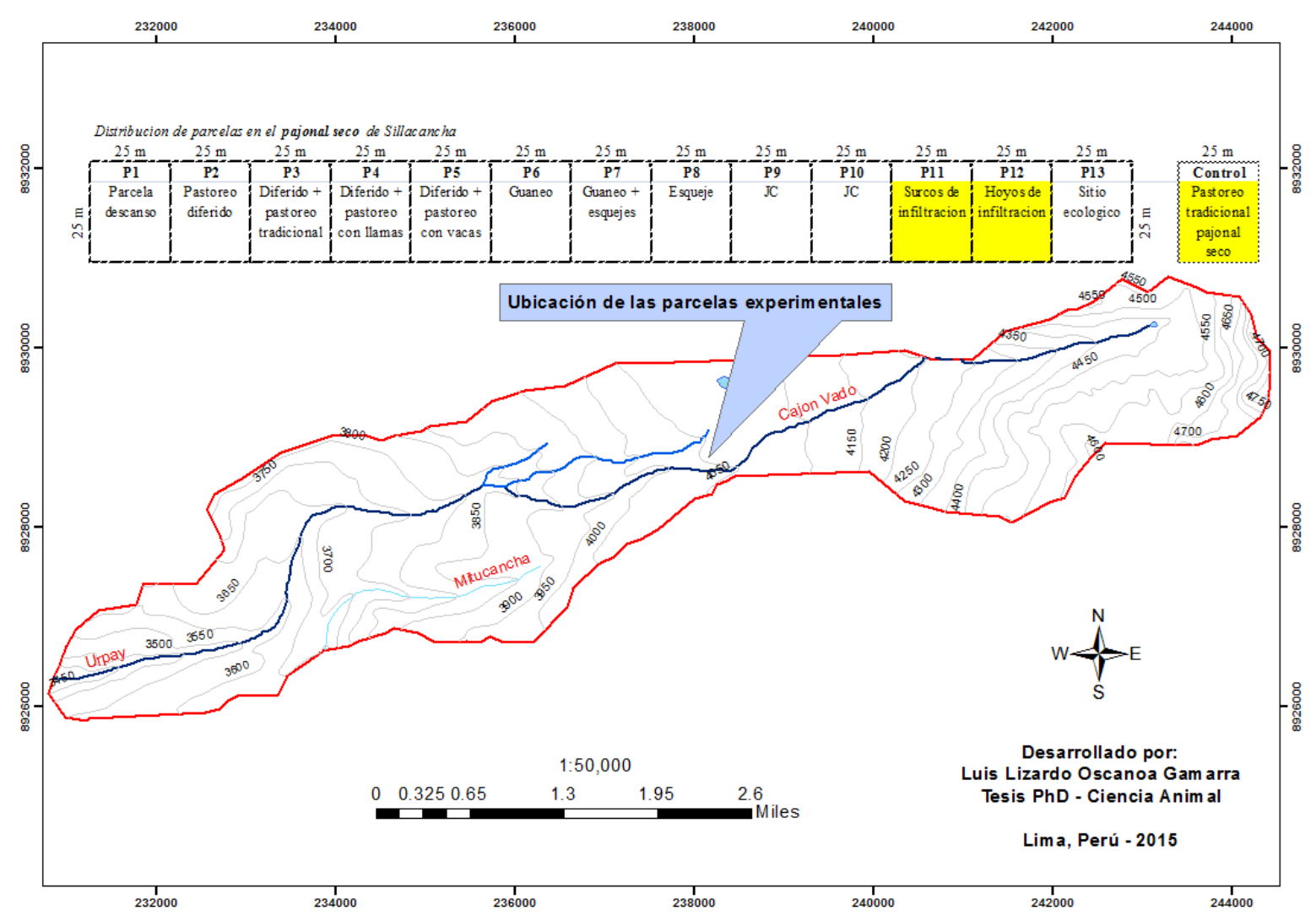

Figura 1: Ubicación de las parcelas experimentales en "Sillacancha”.

Tratamientos Experimentales

Se ensayaron 18 tratamientos producto de la combinación de tres técnicas y seis épocas de muestreo. El monitoreo de los tratamientos se realizó bajo el modelo sistémico aleatorio en parcelas (surcos, hoyos y uso comunal) durante seis épocas de muestreo (may-11, ago-11, oct-11, nov-12, feb-13, set-13). Estos tratamientos fueron estudiados en parcelas cercadas 25 × $25 \mathrm{~m}$, donde los surcos $(15 \mathrm{~cm}$ de ancho por $10 \mathrm{~cm}$ de profundidad) fueron construidos a intervalos de dos metros y los hoyos $(7.5 \mathrm{~cm}$ diámetro por $10 \mathrm{~cm}$ de profundidad) a intervalos de un metro, en referencia al área de pastoreo comunal considerada como testigo (Figura 1, Ecuación 1).

Parámetros Evaluados

El estudio de la condición del pastizal de las parcelas se realizó al inicio y final del experimento a través de los índices de especies deseables (\% D), índice de especies poco deseables (\% A), índice forrajero (IF $=\%$ de especies deseables mas poco deseables), índice suelo desnudo - pavimento de erosión - roca (\% BRP) e índice de vigor de las especies vegetales claves (Parker, 1951). La integración matemática de los índices parciales permitió estimar la condición del pastizal a través de la fórmula: Puntaje $=0.5(\% \mathrm{D})+0.2(\% \mathrm{IF})+$ $0.2(\% \mathrm{COB})+0.1(\% \mathrm{~V})$, donde índice de cobertura $(\mathrm{COB})=100-\%$ BRP. El puntaje total se comparó con la escala puntaje - condición propuesta por Florez et al. (1991).

Para el muestreo del mantillo y biomasa se ubicaron al azar 12 puntos de muestreo. Con la ayuda de un cuadrante de metal de $0.5 \mathrm{~m}^{2}$, se colectó manualmente el tejido vegetal muerto en pie y el recojo cuidadoso de la hojarasca ubicada dentro del cuadrante; en tanto que, para la biomasa, con la ayuda de una tijera de esquila llamada "lapiaco" se cortó toda la fitomasa ubicada dentro del cuadrante. Según las recomendaciones de ASRM (1962) y AOAC $(1980)$ cada muestra fue colocada en bolsa de papel $n^{\circ}$ 12 , se codificaron, se protegieron externamente con bolsa de plástico e inmediatamente se trasladaron al laboratorio de pastizales de la UNALM. En el laboratorio las muestras fueron liberadas de la bolsa de protección, pesadas en balanza de precisión, oreadas, posteriormente se secaron en estufa a temperatura de $105{ }^{\circ} \mathrm{C}$ por 24 horas y las muestras secas pesadas nuevamente en balanza de precisión. Los rendimientos de biomasa y mantillo fueron expresados en $\mathrm{kg} \mathrm{ms} / \mathrm{ha}$.

Se evaluó la temperatura del suelo a profundidad de crecimiento de las raíces $(10-20 \mathrm{~cm})$; para lo cual, luego del muestreo del mantillo y biomasa, se construyeron mini calicatas de $15 \mathrm{~cm}$ de ancho. En cada punto de lectura se esperó cinco minutos para la estabilización del sensor del geotermómetro. Las 
lecturas fueron hechas entre las nueve y once de la mañana debido a la estabilidad de temperatura, viento y baja probabilidad de lluvias que podrían afectar la toma de información.

Para la evaluación de infiltración de suelos, en cada parcela de estudio se seleccionaron dos áreas representativas. Usando una comba y bloque de madera se introdujo el infiltrómetro doble anillo hasta una profundidad aproximada de $10 \mathrm{~cm}$. El infiltrómetro de $2 \mathrm{~mm}$ de espesor fue fabricado en material metálico de medidas de $40 \mathrm{~cm}$ de altura con $40 \mathrm{~cm}$ de diámetro (anillo externo) y $40 \mathrm{~cm}$ de altura con $30 \mathrm{~cm}$ de diámetro (anillo interno). Luego de fijado el infiltrometro en el suelo, se cubrió la parte interna con una capa de plástico con el objeto de amortiguar el golpe del agua al momento del vaciado del agua. Se enrazó el nivel de agua hasta una medida conocida y se dio inicio a la lectura inmediatamente después de liberar el plástico de amortiguación. Las lecturas fueron realizadas en $\mathrm{cm} / \mathrm{min}$ a intervalos de un minuto (cinco lecturas), cinco minutos (cinco lecturas), diez minutos (dos lecturas) hasta encontrar la constancia de las dos últimas lecturas (Bouwer, 1986).

Para el monitoreo de humedad y densidad aparente del suelo (información importante para el cálculo de erosión del suelo), con un cilindro de muestreo (7.62 cm de altura por $7.62 \mathrm{~cm}$ de diámetro) se tomaron muestras de suelo a una profundidad de 10 y $20 \mathrm{~cm}$. Con la ayuda de una comba se introdujo el cilindro en el suelo hasta que toda la cámara del cilindro estuvo completamente llena de suelo, se retiró cuidadosamente el cilindro y con un cuchillo plano se uniformizó la superficie de los extremos del cilindro e inmediatamente cubiertos con papel de hornear, bolsa de plástico, se codificaron y las muestras fueron inmediatamente trasladadas al laboratorio. En el laboratorio, las muestras frescas fueron pesadas, oreadas y secadas en estufa a $105{ }^{\circ} \mathrm{C}$ por 24 horas. Luego de este tiempo, las muestras secas fueron pesadas en balanza de precisión y los resultados expresados en porcentaje (Blake, 1965).

Para la evaluación del control de la erosión del suelo, se colocaron en la parte alta de la parcela una fila de cuatro varillas y otra fila de cuatro varillas en la parte inferior de la parcela a intervalos de $5.25 \mathrm{~m}$ entre varilla y varilla. Las varillas fueron redondas de un diámetro de $4 \mathrm{~mm}$ y $40 \mathrm{~cm}$ de largo las que fueron fijadas en el suelo a una profundidad de $25 \mathrm{~cm}$. Las lecturas fueron realizadas con vernier digital a cuatro lados de la varilla (superior, inferior, derecha e izquierda). Los datos fueron sumados algebraicamente respetando los signos de los resultados, de manera que se consideró a los signos positivos $(+)$ como perdida del suelo y a los negativos (-) como acumulación de suelo. Para el cálculo de la pérdida del suelo se usó la fórmula (Vásquez \& Tapia, 2011):

$$
\mathrm{Ps}=\mathrm{h} * \text { Dap } * 10
$$

Donde:

Ps = Pérdida de suelo por erosión o sedimentación (ton/ha).

h = Lámina de suelo erosionado o sedimentado (mm).

Dap = Densidad aparente del suelo (gr/cm3).

\section{Diseño Experimental.}

Se empleó el Diseño Completamente al Azar con arreglo factorial con dos factores A (dos técnicas de mejora y testigo) y B (seis meses de muestreo), donde los tratamientos considerados fueron la interacción de las técnicas con los meses de muestreo, cuyas combinaciones hicieron un total de 18 tratamientos. El Diseño Factorial fue no balanceado (desigual cantidad de observaciones por casilla) y fue completo, ya que se realizaron todas las posibles combinaciones entre los valores de las variables.

Modelo Estadístico:

Ecuación 1

$$
\begin{aligned}
& Y_{i j k}=\mu+\alpha_{i}+\tau_{j}+\alpha \tau_{i j}+e_{i j k} \\
& i=1,2,3 ; j=1,2,3,4,5,6 ; \quad k=1,2 \ldots ., n_{i j}
\end{aligned}
$$
donde:

$\boldsymbol{Y}_{i j k}=$ Es la respuesta obtenida (rendimiento de los parámetros hidrológicos del pastizal) en la unidad experimental correspondiente a la k-ésima repetición a la cual se le aplicó el i-ésimo nivel del factor A, con el j-ésimo nivel del factor B.

$\mu=$ Efecto de la media general.

$\alpha_{i}=$ Efecto del i-ésimo nivel del factor técnica de mejora (A).

$\tau_{j}=$ Efecto del j-ésimo nivel del factor época de muestreo (B).

$\alpha \tau_{i j}=$ Efecto de la interacción entre el i-ésimo nivel del factor A con el j-ésimo nivel del factor B.

$\boldsymbol{e}_{i j k}=$ Variación del error asociado con las ijk unidades.

El análisis de medias midió la intensidad de la relación entre las variables, las asociaciones entre las categorías mediante la producción de dimensiones representativas de las distancias chi-cuadrado y proporcionó diversos marcos de interpretación a los gráficos, en términos de distancias, centroides, modelos de asociación, y chi-cuadrado (Montgomery, 1991). Para el análisis de los promedios de los 
parámetros estudiados se utilizó la prueba de medias de Duncan $(\mathrm{P}<0.05)$.

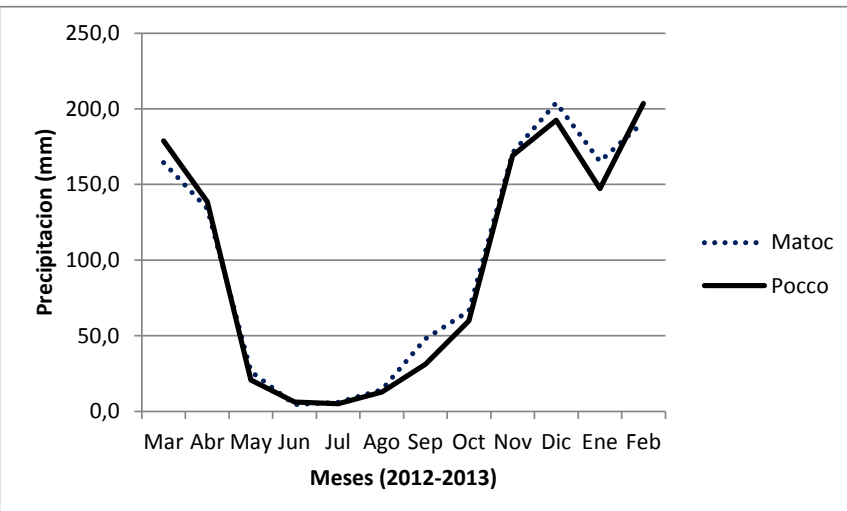

Figura 2: Curva de precipitación del área experimental "Sillacancha". del pastizal es un diagnóstico semidetallado o detallado de los cambios que han ocurrido en la cobertura vegetal $y$ de esta forma provee información de análisis para predecir la naturaleza y dirección de los cambios en la comunidad vegetal por la utilización de recursos y otras acciones; conceptualmente, las clases de condición son la expresión del grado en que la composición florística se ha modificado en relación a la comunidad clímax. Dentro de este contexto, la composición florística y precipitación varían por época del año y la mayor cantidad de precipitación se presenta durante los meses diciembre - febrero, y la menor durante junio - agosto; eventos que coinciden con el comportamiento de producción de fitomasa e indican que a menor producción menor infiltración (Bari et al., 1993).

Existe una interacción significativa del factor técnica por época del año en la variable biomasa

\section{Resultados y discusión.}

Estado Ecológico del Pastizal.

A dos años de estudio, el estado ecológico del pastizal mantuvo el calificativo de muy pobre (para ovinos, vacunos y vicuñas) al aplicarse las técnicas de surcos y hoyos dentro un marco estacional de lluvias (Tabla 1, Figura 2). Si bien en la comunidad vegetal continua es dominante la especie Calamagrostis macrophylla, en la categoría sub-sub-dominante se observó la recuperación de especies vegetales perennes catalogadas como indicadoras de cambios de humedad del suelo (surcos: SCIRI, hoyos: CAVI) en comparación al testigo que continúa exhibiendo plantas anuales (MUPE). Estos resultados evidencian que el diagnóstico de la condición del pastizal requiere ser complementado con otros indicadores más sensibles y precisos para el desarrollo sustentable de programas de manejo ecohidrológico del pastizal.

Flores y Bryant (1989) indicaron que la condición

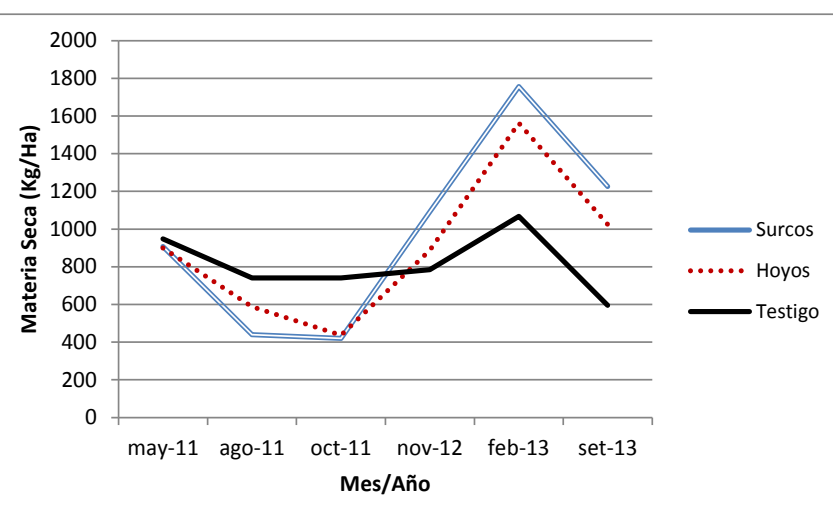

Figura 3: Curva temporal de fitomasa aérea del pastizal tratado con técnicas de mejora de suelos.

$(\mathrm{p}=0.001<0.05)$. Los surcos, alcanzaron niveles bajos al inicio del experimento, y luego, conforme se cicatrizó la superficie del suelo, esta situación se mostró superior al de los hoyos y al testigo a partir de

Tabla 1: Condición del pastizal de las parcelas experimentales "Sillacancha".

\begin{tabular}{|c|c|c|c|c|c|c|c|}
\hline \multirow{2}{*}{ Especie vegetal } & \multirow{2}{*}{$\begin{array}{l}\text { Especie } \\
\text { animal y } \\
\text { rendimiento }\end{array}$} & \multicolumn{2}{|c|}{ Surcos } & \multicolumn{2}{|c|}{ Hoyos } & \multicolumn{2}{|c|}{ Testigo } \\
\hline & & Oct-11 & Sep-11 & Oct-11 & Sep-11 & Oct-11 & Sep-11 \\
\hline Dominante & & Cama & Cama & Cama & Cama & Cama & Cama \\
\hline Sub-dominante & & Sciri & Sti sp & Sti sp & Acpu & Sciri & Sciri \\
\hline Sub-sub-dominante & & Mupe & Sciri & Mupe & Cavi & Plan sp & Mupe \\
\hline \multirow{3}{*}{$\begin{array}{l}\text { Puntaje ponderado y } \\
\text { condición del pastizal }\end{array}$} & Ovinos & $12.5 \mathrm{Mp}$ & $12.5 \mathrm{Mp}$ & $16.4 \mathrm{Mp}$ & $16.6 \mathrm{Mp}$ & $12 \mathrm{Mp}$ & $12.5 \mathrm{Mp}$ \\
\hline & Vacunos & $12.5 \mathrm{Mp}$ & $12.5 \mathrm{Mp}$ & $14.9 \mathrm{Mp}$ & 16.1 Mp & $12 \mathrm{Mp}$ & $12.5 \mathrm{Mp}$ \\
\hline & Vicuñas & $12.5 \mathrm{Mp}$ & $10 \mathrm{Mp}$ & $18.4 \mathrm{Mp}$ & 17.1 Mp & $10 \mathrm{Mp}$ & $9 \mathrm{Mp}$ \\
\hline Biomasa & kgms/ha & 419.7 & 1226.1 & 436.5 & 1025.6 & 741.2 & 595.7 \\
\hline Mantillo & $\mathrm{kgms} / \mathrm{ha}$ & 105.2 & 161.1 & 203.2 & 141.2 & 135.5 & 112.4 \\
\hline \multicolumn{4}{|c|}{ CAMA: Calamagrostis macrophyla } & \multicolumn{4}{|c|}{ CAVI: Calamagrostis vicunarum } \\
\hline \multicolumn{4}{|l|}{ SCIRI: Scirpus rigidus } & \multicolumn{4}{|c|}{ PLAN sp: Plantagon sp. } \\
\hline \multicolumn{4}{|c|}{ STI sp: Stipa sp. } & \multicolumn{4}{|c|}{ MUPE: Muhlenbergia peruviana } \\
\hline \multicolumn{4}{|c|}{ ACPU: Aciachne pulvinata } & \multicolumn{4}{|c|}{ MP: Muy pobre. } \\
\hline
\end{tabular}


noviembre 2012 (Figura 3). El mejor rendimiento de biomasa se produjo en febrero $2013(\mathrm{p}=0.029<0.05)$ en clara coincidencia con la mayor precipitación ocurrida en la zona (Figura 2), y que en promedio la mayor producción se registró con el aplicativo surco que los hoyos y el testigo (1226, 1026, 596 kg $\mathrm{ms} /$ ha, respectivamente).

Estudios sobre el particular encontraron efectos positivos de la cosecha del agua por las plantas de los pastizales en términos de cantidad $\mathrm{y}$ calidad (Elsadig et al., 2013); y que la composición y rendimiento de los pastizales, durante la estación de crecimiento, está influenciada por la disponibilidad de agua, nutrientes del suelo y pastoreo; éste último altera los ciclos del agua y nutrientes, a través de la defoliación y reducción de residuos vegetales que favorece la presencia de especies tolerantes menos productivas (Willmst \& Jefferson, 1990). Argumentos que explican el por qué la negativa tendencia del rendimiento de biomasa en el territorio de uso comunal.

Existe un efecto significativo del factor época del año en la variable mantillo $(\mathrm{p}=0.000<0.05)$. El rendimiento del mantillo de las parcelas con surcos y hoyos, tuvieron el mismo comportamiento estacional que el rendimiento de biomasa y la curva de precipitación (Figuras 2, 3 y 4). En el periodo febrero 2013 la producción del mantillo mostró mejor nivel de producción que los otros meses $(\mathrm{p}=0.029<0.05)$; y en promedio las tendencias de valores se mostraron favorables para el aplicativo de surcos que para hoyos y el testigo (161, 141, $112 \mathrm{~kg} \mathrm{~ms} / \mathrm{ha}$, respectivamente).

La idoneidad del suelo para sostener el crecimiento de plantas y la actividad biológica está en función a las propiedades físicas y químicas, muchas de las cuales dependen de la cantidad y calidad del mantillo; el cual aumenta significativamente la disponibilidad del agua, porosidad total y retención de la humedad del suelo y muestra alta correlación con la estabilidad de agregados (Nagaya \& Lal, 2008).

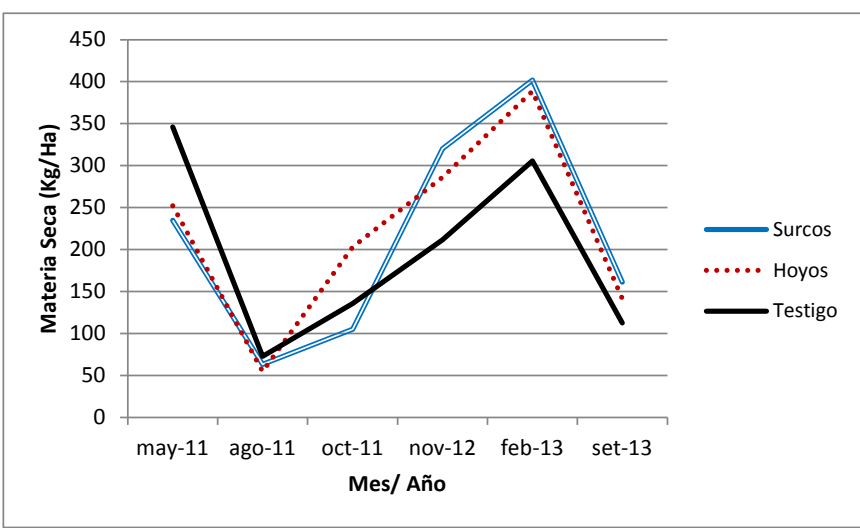

Figura 4: Curva temporal del mantillo del pastizal tratado con técnicas de mejora de suelos.

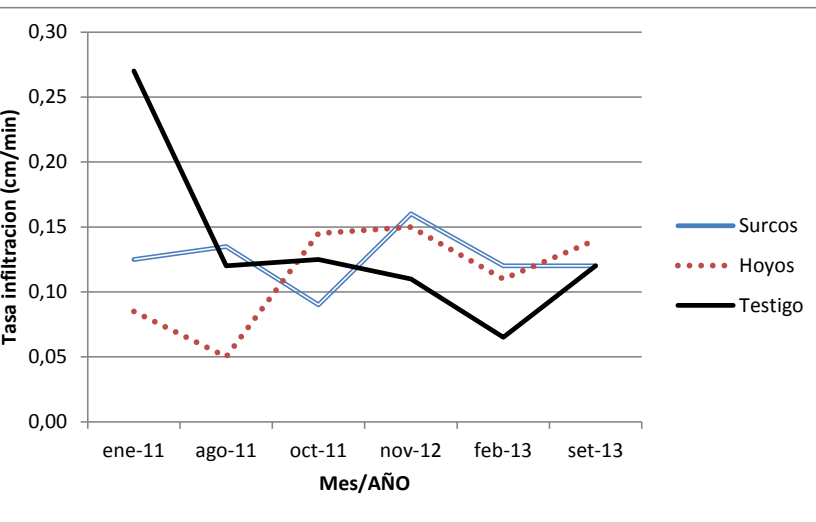

Figura 5: Curva temporal de la infiltración del suelo del pastizal tratado con técnicas de mejora de suelos.

En el prado seco, con mayor humedad del suelo, se encontró mayor respuesta de producción al nitrógeno (nitrógeno-fosforo), a pesar de las respuestas funcionales de las especies vegetales dominantes (William et al., 1993). Otros estudios han encontrado que el mantillo tiene mayor impacto después de la senescencia de la planta y antes del período de crecimiento de la planta (Walter \& Peterson, 1990); y que en pradera seca, la producción máxima se alcanza con la máxima cantidad de mantillo, por lo que el pastoreo debe manejarse conservando el mantillo con el fin de estabilizar la producción ganadera y mantener la condición del pastizal (Willms et al., 1993).

Respuesta Hidrológica del Pastizal.

Existe una interacción significativa del factor técnica por época del año en la variable tasa de infiltración $(\mathrm{p}=0.001<0.05)$. El comportamiento de las curvas de las medias marginales de infiltración sugiere superioridad sostenida de los surcos y hoyos a partir del periodo octubre 2011 en comparación al testigo (Figura 5). La máxima infiltración ocurrió durante el periodo febrero $2013(\mathrm{p}=0.001<0.05)$; y en promedio, la evolución de la tasa de infiltración del periodo setiembre $2013(0.127 \mathrm{~cm} / \mathrm{min})$ fue menor que en noviembre $2012(0.140 \mathrm{~cm} / \mathrm{min})$ y enero $2011(0.160$ $\mathrm{cm} / \mathrm{min}$ ) nos sugiere mayor retención de agua de lluvias infiltrada en concordancia al incremento del mantillo en el suelo (Figura 4).

Pastizales de condición muy pobre con técnicas de mejora de suelos muestran recuperación de niveles de infiltración, lo cual coincidió con las investigaciones de Chartier et al. (2011) quienes hallaron que la vegetación e infiltración del suelo están estrechamente acopladas, y que el pastoreo animal afecta tanto a los procesos ecológicos e hidrológicos, así como a sus interacciones. Fiedler et al. (2002) en sus investigaciones encontraron una relación estrecha entre la micro topografía y la comunidad vegetal de los pastizales e indicaron que las interacciones de la superficie del suelo fueron un componente importante de los efectos 


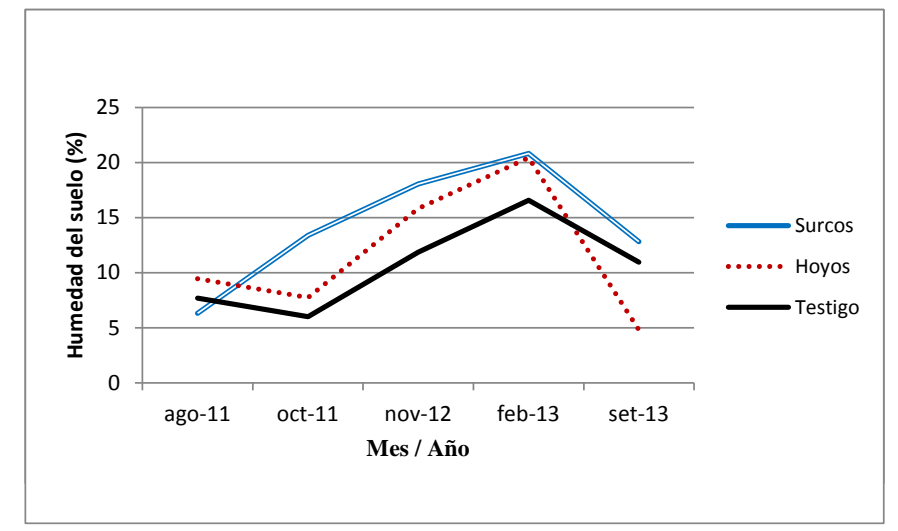

Figura 6: Curva temporal de la humedad del suelo del pastizal tratado con técnicas de mejora de suelos.

de pastoreo sobre la respuesta hidrológica de los pastizales debido al sellado de la superficie desnuda del suelo.

Existe una interacción significativa del factor técnica por época del año en la variable humedad del suelo $(p=0.001<0.05)$. Las curvas de las medias marginales muestran superioridad, durante el periodo noviembre 2012 y febrero 2013, favorable al aplicativo surco que hoyos y uso comunal $(p=0.000<0.05)$ (Figura 6). Para el caso particular de los hoyos, al final del experimento, se observó declive abrupto del porcentaje de humedad del suelo, el cual podría deberse a que estos se llenaron nuevamente de material arrastrados por caída de lluvias (Figura 2).

En promedio los niveles más altos de biomasa y humedad del suelo se dieron en la época de lluvias del segundo año de estudio (334 mm/mes), estos sustentan el efecto positivo de la precipitación sobre el desarrollo de la planta, lo que confirmaría una significativa influencia de las técnicas de conservación de suelos sobre el contenido de humedad del suelo. Los resultados fueron explicados por Snymana \& Du Preez (2005) quienes encontraron que los cambios en los niveles de humedad del suelo variaron en concordancia al grado de degradación del suelo. De manera que la precipitación variada afecta la cantidad de agua que penetra en el suelo y siendo la lluvia la única fuente de recarga de agua en el pastizal, la textura, cubierta vegetal, y elevación aumentaron significativamente la cantidad de humedad del suelo retenido (Salve \& AllenDiaz, 2001).

Existe un efecto significativo del factor época del año en la variable temperatura $(p=0.000<0.05)$. Las curvas de las medias marginales estimadas de la temperatura del suelo muestran que la época de mayor efecto fue durante el periodo setiembre 2013 $(p=0.000<0.05)$ (Figura 7). En tanto, que las medias de los estadísticos descriptivos muestran una superioridad favorable para surcos $(11.00$ $\left.{ }^{\circ} \mathrm{C}\right)$ que hoyos $\left(10.67^{\circ} \mathrm{C}\right)$ y el testigo $\left(10.00^{\circ} \mathrm{C}\right)$.
Funcionalmente, los beneficios de esta mejora son para la resiembra natural de las especies nativas, ya que a temperaturas de confort se activan todos los procesos germinativos, y permiten mayor posibilidad de establecimiento de las plantas tiernas.

Los valores promedios de los hoyos, al segundo año de investigación (setiembre 2013), nos sugieren que estas se van acercando a condiciones adecuadas para el proceso biológico; el cual es superior a $15^{\circ} \mathrm{C}$ (Wildung et al., 1975). En este contexto, la producción del ecosistema pastizal se encuentra influenciado por la composición florística y los microbios del suelo, que interactúan con el ambiente que les rodea; factores del suelo, como la temperatura, humedad, y sustrato influyen simultáneamente en el rendimiento vegetal (Raich \& Tufekcioglu, 2000).

Maher et al. (2010) encontraron un aumento significativo de la respiración en pradera vieja y una correlación positiva con la biomasa de raíces y la productividad primaria neta durante el crecimiento del pastizal, e indicaron que la temperatura del suelo es un fuerte predictor de la respiración intra-estacional entre praderas. De manera que la humedad del suelo fue el factor ambiental dominante que controló la variación estacional e interanual de la respiración del prado a temperatura constante de $10^{\circ} \mathrm{C}$ (Lawrence \& Bruce, 2005), lo que implicó mayor producción de biomasa en las parcelas con prácticas de conservación "surcos” y "hoyos".

Control de la Erosión del Suelo.

Existe un efecto significativo del factor época del año en la variable control de la erosión del suelo $(p=0.003<0.05)$. Las curvas de las medias marginales muestran que en el periodo junio 2012, en la parcela testigo, se produce una significativa variación en comparación a los surcos y hoyos que son menos pronunciadas $(p=0.002<0.05)$ (Figura 8). El total acumulado (diciembre 2011 a setiembre 2013) indica que hay mejor control de pérdida de suelos con la técnica de surcos (36 tm/ha) y hoyos (45 tm/ha) que el

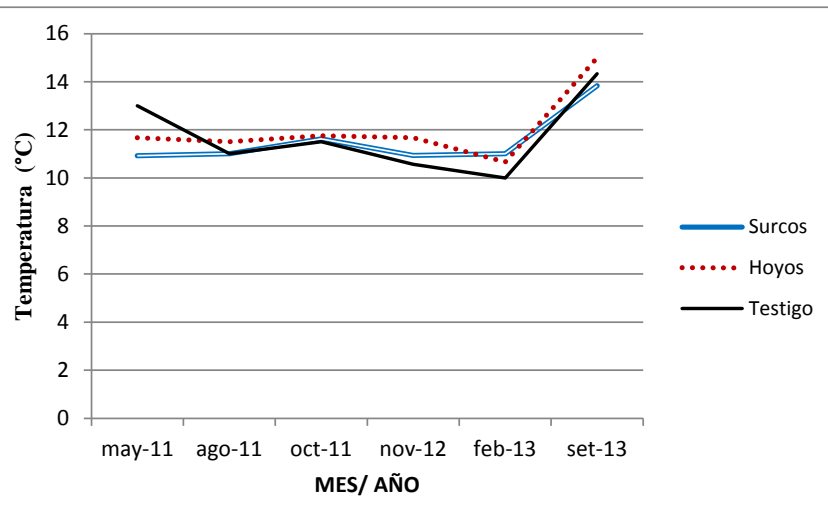

Figura 7: Curva temporal de temperatura del suelo del pastizal tratado con técnicas de mejora de suelos. 
testigo (58 tm/ha). Debido a que los pastizales son destinados únicamente para el pastoreo animal, supondría que el inadecuado movimiento animal fue el factor determinante para el disturbio del suelo y la mayor irregularidad del movimiento del suelo.

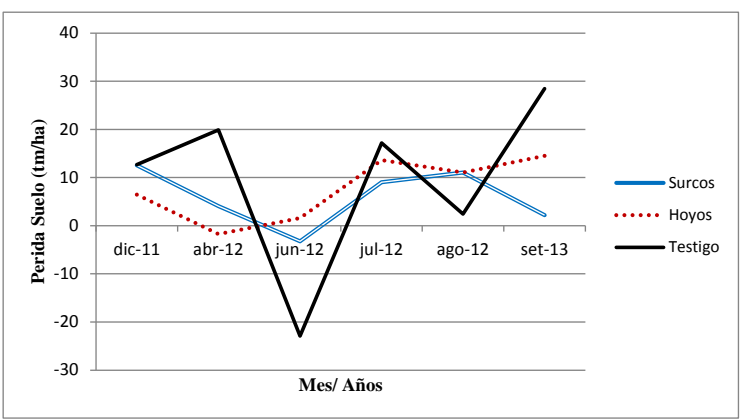

Figura 8: Curva temporal de la erosión del suelo del pastizal tratado con técnicas de mejora de suelos.

Los resultados encontrados son relativamente menores a los registrados por Vásquez \& Tapia (2011) quienes indicaron que la pérdida de suelo, en laderas de la sierra peruana, fue sumamente alta (45 ton/ha/año o $3.20 \mathrm{~mm} / \mathrm{año)} \mathrm{y} \mathrm{cuando} \mathrm{éstas} \mathrm{fueron}$ tratadas con zanjas de infiltración se redujo la perdida en aproximadamente a mitad del testigo (24.44 ton/ha/año o $1.73 \mathrm{~mm} / \mathrm{año})$. Sobre el problema, Zhou et al. (2010) señalaron que, si bien es cierto que los animales al pastoreo son medio de vida para los agricultores, en la práctica estos causan efectos negativos por pisoteo animal con efectos graves sobre la compactación del suelo que dañan las propiedades físicas del suelo, que a la larga incrementan la erosionabilidad de la tierra vegetal. A la luz de estos resultados los surcos y hoyos manifiestan ser técnicas de mínimo costo y uso de recursos para la conservación ecohidrologica del pastizal dedicadas a la producción de animales al pastoreo.

\section{Conclusiones.}

Los resultados de la investigación demostraron que la introducción de técnicas de surcos y hoyos posibilita mejorar el estado ecológico del pastizal de condición muy pobre con menor uso de tiempo y recursos; así lo sustenta la recuperación de la especie vegetal Scirpus rigidus (planta nativa indicadora de pulsos de humedad del suelo), e incremento del rendimiento de biomasa y mantillo del área experimental dominado por la Calamagrostis macrophylla. El estudio revela que la implementación de surcos y hoyos coadyuva a recuperar las facultades hidrológicas del pastizal degradado, probablemente como resultado de la mejora de captación del agua de lluvias, cobertura vegetal, mantillo, humedad del suelo y disminución de la evapotranspiración de la comunidad vegetal; por lo que su elección y monitoreo participativo fue lo más pertinente como estrategia cooperativa para la mejora del pastizal degradado. Como opción técnica los surcos y hoyos demostraron mejor control de la erosión del suelo que el testigo. La investigación proporciona evidencia de la importancia de profundizar en estudios ecohidrológicos de los tipos de pastizal en deterioro y la necesidad de generar propuestas, de menor costo de tiempo y dinero, alrededor de las técnicas de mejora estudiadas que son la base promisoria para la reintroducción de las especies vegetales de importancia hidrológica del ecosistema pastizal degradado.

\section{Agradecimientos.}

Al Comité de Agropecuaria Local “Alli Pastu” de Canrey Chico - Huaraz, a The Mountain Institute y al Laboratorio Utilización de Pastizales de la Universidad Nacional Agraria La Molina por el apoyo técnico; a The Collaborative Crop Research Program/ The MckNight Foundation por la beca doctoral y al Ph.D. Jorge Recharte, Ph.D. Javier Ñaupari e Ing. José Haro por los comentarios al contenido del presente artículo.

\section{Literatura citada.}

American Society of Range Management (ASRM). 1962. Basic problems and techniques in range research. A report of a joint committee of the ASRM and Agriculture board. Publication $\mathrm{N}^{\circ}$ 890. Washington D.C. 45-81 pp.

Association of Official Analytical Chemist (A.O.A.C.) 1980. Official methods of analysis $\left(13^{\text {th }} \mathrm{ed}\right)$. Washington D.C. 125 pp.

Bari F., Wood k. \& Murray L. 1993. Livestock grazing impacts on infiltration rates in a temperature range of Pakistan. J. Range Manage. 46: 367-372.

Blake G. R. 1965. Methods of Soil Analysis, Part 1. Physical and Mineralogical Properties, Including statistics of Measurement and Sampling. ASA-SSSA, Agronomy Monograph 9: 374-390.

Bouwer H. 1986. Intake rate: cylinder infiltrometer, Methods of Soil Analysis, Part 1: Physical and Mineralogical Methods, 2nd Ed. Madison: American Society of Agronomy and Soil Science Society of America. 825-844.

Bork E.W., Thomas T. \& Mcdougall B. 2001. Herbage response to precipitation in central Alberta boreal grasslands. J. Range Manage. 54: 243-248.

Breshears D.D. \& Barnes F.J. 1999. Interrelationships between plant functional types and soil moisture heterogeneity for semiarid landscapes within the grassland/forest continuum: a unified conceptual model. Landscape Ecology 14: 465-478.

Bryant D.M., Holland E.H., Seastedt T.R. \& Walker M.D. 1998. Analysis of litter decomposition in an alpine tundra. Can. J. Bot. 76: 1295-1304.

Chartier M.P., Rostagno C.M. \& Pazos. 2011. Effects of soil degradation on infiltration rates in grazed semiarid rangelands of northeastern Patagonia, Argentina. Journal of Arid Environments 75: 656 - 661.

Eekeren N.V., Boer H., Hanegraaf M., Bokhorst J., Nierop D., Bloem J., Schouten T., Goede R., \& Brussaard L. 2010. Ecosystem services in grassland associated with 
biotic and abiotic soil parameters. Soil Biology \& Biochemistry 42:1491-1504.

Elsadig A., Saidi S., Adeeb A. \& Ickowicz A. 2013. Water Harvesting Model for Improved Rangeland Productivity. International Journal of Water Resources and Arid Environments 2(2): 64-77.

Eviner V. 2004. Plant traits that influence ecosystem processes vary independently among species. Ecology, 85(8): 2215-2229.

Fiedler F.R., Frasier G.W., Ramirez J.A. \& Ahuja L.R. 2002. Hydrologic Response of Grasslands: Effects of Grazing, Interactive Infiltration, and Scale. Journal of Hydrologic Engineering, July-August. 293-301.

Florez A. \& Bryant E. 1989. Manual de pastos y forrajes. Programa colaborativo de apoyo a la investigación en rumiantes menores. INIIA, Universidad de california. Lima - Perú.

Florez A. \& Malpartida E. 1987. Manejo de praderas nativas y pasturas en la región altoandina del Perú. Banco Agrario, Tomo I. Lima Perú. 329 pp.

Florez M.A., Malpartida I.E. \& San Martin, F. 1991. Manual de forrajes para zonas áridas y semiáridas andinas. 308 pp.

Getahun S.Y., Tobias P., Schweitzer C., Lierschb S., Van Der J.K., Marloes L.M., Van A.G. \& Vander P.Z. 2014. Dynamic feedback between land use and hydrology for ecosystem services assessment: International environmental modeling and software sociery (iEMSs) $7^{\text {th }}$ Intl. Congress on Env. Modeling and software, San Diego: CA: USA, Daniel P.Ames, Nigel W.T. Quinn and Andrea E. Rizalli (Eds).

Gil R.J. 2014. Monitoreo hidrológico en ecosistemas de la puna húmeda bajo el método de cuencas pareadas: C.C. Cordillera Blanca, Canrey Chico, Recuay, Ancash, Perú. Proyecto Puna-agua. Conv. Instituto de Montaña/ The MckNight Foundation. Documento en prensa. 49 p.

Holdridge L.R. 1987. Ecología basada en las zonas de vida. Instituto Interamericano de Cooperación para la Agricultura (IICA). San José de Costa Rica. 219 pp.

Laboratorio de Análisis de Suelos y Aguas UNALM. 2011. Resultados de caracterización físico-químico de suelos.

Lawrence B.F. \& Bruce J. 2005. Interacting effects of temperature, soil moisture and plant biomass production on ecosystem respiration in a northern temperate grassland. Agricultural and Forest Meteorology 130: 237-253.

Ma W., Liu Z., Wang Z., Wang W., Liang C., Tang Y., He JS., \& Fang J. 2010. Climate change alters interannual variation of grassland aboveground productivity: evidence from a 22-year measurement series in the Inner Mongolian grassland. J Plant Res. 123:509-517.

Maher R., Asbjornsena H., Kolka R., Cambardellac A. \& Raichd, J. 2010. Changes in soil respiration across a chronosequence of tallgrass prairie reconstructions. Agriculture, Ecosystems and Environment 139: 749753.
Montgomery D.C. 1991. Diseño y análisis de experimentos. Arizona State University, Estados Unidos de América. Grupo Editorial Iberoamérica. 589 pp.

Nagaya M. \& Lal R. 2008. Mulching effects on selected soil physical properties. Soil \& Tillage Research. 98: 106111.

Parker K. W. 1951. A method for measuring trend and range condition on National Forest Ranges. USDA. Forest Sevice. 26 pp.

Raich W.J. \& Tufekcioglu A. 2000. Vegetation and soil respiration: Correlations and controls. Biogeochemistry 48: 71-90.

Salve R. \& Allen-Diaz B. 2001. Variations in soil moisture content in a rangeland catchment. J. Range Manage. 54: 44-51.

Snymana H. \& Preez D. 2005. Rangeland degradation in a semi-arid South Africa-II: influence on soil quality. Journal of Arid Environments 60: 483-507.

Walter P. \& Peterson J. 1990. The role of litter in an oldfield community: impact of litter quantity in different seasons on plant species richness and abundance. Oecologia, 85: 8-13.

Wildung R.E., Garland T.R. \& Buschbom R.L. 1975. The interdependent effects of soil temperature and water content on soil respiration rate and plant root decomposition in arid grassland soils. Soil Biology and Biochemistry, Volume 7: 373-378.

William D., Theresa A., James C. \& Conant T. 1993. Constraints of nutrient availability on primary production in two alpine tundra communities. Ecology, 74(7): 2085-2097.

Willms D.W. \& Jefferson P.G. 1990. Production characteristic of the mixed praire: constraints and potential. Can. J. Anim. Sci. 73t 765-778.

Willms D.W., Mcginn M.S., \& Dormaar F. 1993. Influence of litter on herbage production in the mixed prairie. J. Range Manage. 46: 320-324.

Vásquez A. \& Tapia M. 2011. Cuantificación de la erosión hídrica superficial en las laderas semiáridas de la sierra peruana. Rev. Ingeniería UC, Vol.18, No 3. 42-50.

Viramontes-Olivas O. A., Reyes-Gómez V.M., RangelRodríguez A., Ortega-Ochoa C. Soto-Cruz, R.A., Camarillo J.A. \& T. Lebgue-Keleng. 2012. Papel hidrológico-ambiental de pastizales nativos e introducidos en la cuenca alta del río Chuvíscar, Chihuahua, México. TECNOCIENCIA Chihuahua 6(3): 181-193.

Zhang, Y., Hernandez, M., Anson, E., Nearing, M.A., Wei, M.A., Stone, J.J. \& P. Heilman. 2012. Modeling climate change effects on runoff and soil erosion in southeastern Arizona rangelands and implications for mitigation with conservation practices. Journal of Soil and Water Conservation. 67 (5): 390 - 405.

Zhou Z.C., Gan Z.T., Shangguan Z.P. \& Dong Z.B. 2010. Effects of grazing on soil physical properties and soil erodibility in semiarid grassland of the Northern Loess Plateau (China). Catena 82: 87-91.

\footnotetext{
${ }^{1}$ Ph.D. (c) Investigador asociado, The Mountain Institute - Laboratorio Pastizales UNALM, Lima Perú.

${ }^{2}$ Ph.D. Manejo de Pastizales, Profesor Principal EPG UNALM, Lima Perú.
} 